\title{
BIOLOGIA ALIMENTAR DE STEINDACHNERINA BREVIPINNA (CHARACIFORMES, CURIMATIDAE) DO RIO IBICUÍ-MIRIM, RIO GRANDE DO SUL, BRASIL
}

\author{
Júlia Giora ${ }^{1}$ \\ Clarice B. Fialho ${ }^{1}$
}

\begin{abstract}
FEEDING BIOLOGY OF STEINDACHNERINA BREVIPINNA (CHARACIFORMES, CURIMATIDAE) IN THE IBICUÍ-MIRIM RIVER, RIO GRANDE DO SUL, BRAZIL. The feeding biology of Steindachnerina brevipinna (Eigenmann \& Eigenmann, 1889) is described based on specimens collected in the Ibicuí-Mirim river, Rio Grande do Sul, Brazil, from April 2001 to March 2002. Diet description is based on the analysis of the frequency of occurrence and index of dietary importance of the ingested items. The monthly variation of stomach repletion, hepatosomatic and intestinal indexes was also analyzed. Major feeding activity occurred before (May, June and July 2001) the reproductive period, with the hepatosomatic index values showing similar variation along the year. Both the mean intestinal quotient and the analysis of the main ingested items (Bacillariophyta and Chlorophyta algae) indicate a detritivorous diet.
\end{abstract}

KEYWORDS. Detritivory, feeding, Steindachnerina, fishes.

\section{INTRODUÇÃO}

A família Curimatidae compreende diversas espécies popularmente conhecidas como birus, extremamente abundantes e de importância ecológica nas comunidades de peixes neotropicais, por serem animais de hábito detritívoro. São peixes de pequeno porte, não apresentam dentes e vivem agrupados próximo ao fundo, em águas abertas (FINK \& FINK, 1978). Muitas espécies neotropicais consomem detrito: algumas são consumidoras ocasionais, enquanto outras, tais como espécies das famílias Prochilodontidae, Curimatidae e Loricariidae, são altamente especializadas (BowEN, 1984). Segundo DARNELL (1964), detrito orgânico é todo tipo de material biogênico em vários estágios de decomposição microbiana, consistindo em um dos mais importantes recursos alimentares e uma das principais vias de ciclagem de matéria orgânica em ecossistemas límnicos. Embora muitas evidências apontem para isso, até o momento não foi demonstrada claramente a significância nutricional do detrito orgânico na economia das comunidades aquáticas (DARNELL, 1961).

1. Laboratório de Ictiologia, Departamento de Zoologia, Programa de Pós-Graduação em Biologia Animal, Universidade Federal do Rio Grande do Sul, Av. Bento Gonçalves, 9500, Prédio 43435, 90540-000 Porto Alegre, RS, Brasil. 
Segundo RoberTs (1972) e Goulding (1980), o comportamento alimentar de peixes da família Curimatidae é, possivelmente, mais complexo que o presumido pela sua categoria trófica, podendo ocorrer inclusive partilha de recursos entre estes peixes detritívoros, embora a natureza do alimento aparentemente impeça uma escolha refinada dos itens. A espécie Steindachnerina brevipinna (Eigenmann \& Eigenmann, 1889) tem ocorrência restrita à América do Sul, distribuindo-se nos rios Paraguai, baixo Paraná e baixo Uruguai, onde apresenta-se muito abundante, ocupando papel de importância na cadeia trófica. $\mathrm{O}$ objetivo é analisar qualitativamente a dieta, estimar os índices de repleção e hepatossomático e calcular o quociente intestinal da espécie.

\section{MATERIAL E MÉTODOS}

A área de estudo compreende o rio Ibicuí-Mirim (2950'14”S e 5447’53”W), na divisa entre os municípios de Cacequi e São Vicente do Sul. Este rio é um tributário do rio Ibicuí, pertencente à bacia do rio Uruguai, que é um dos principais sistemas de drenagem do Estado do Rio Grande do Sul. O trecho amostrado apresenta fundo arenoso, com correnteza de média a rápida e vegetação arbustiva abundante na orla. A distância entre as margens mostrou-se muito instável ao longo das amostragens, variando aproximadamente entre 10 e mais de 30 metros, acompanhando a flutuação pluviométrica. $\mathrm{Na}$ maioria dos meses, essas bordas apresentaram-se praticamente retas, sem recortes. Nos meses de cheia, com a invasão de outros ambientes pelas águas do rio, formaram-se algumas áreas de remansos marginais.

Foi examinado um total de 315 indivíduos, 198 fêmeas e 117 machos, com variação de comprimento de 33,61 a $125,00 \mathrm{~mm}$. As coletas foram realizadas mensalmente, de abril de 2001 a março de 2002, utilizando redes do tipo picaré. Os indivíduos coletados foram fixados em formol $10 \%$ e posteriormente conservados em álcool $70 \%$. Espécimens foram depositados no Departamento de Zoologia, Universidade Federal do Rio Grande do Sul (UFRGS 5244). No laboratório, foram registrados os dados de comprimento total $(\mathrm{mm})$, comprimento do intestino $(\mathrm{mm})$, peso total do estômago e do fígado, e determinados os estádios de repleção do estômago: vazio, parcialmente vazio, parcialmente cheio e cheio. $\mathrm{O}$ índice de repleção (IR) foi estabelecido através da relação percentual entre o peso do estômago e o peso total do peixe, e o índice hepatossomático (IHS), por meio do quociente entre o peso do fígado e o peso total. O quociente intestinal foi determinado através da razão entre o comprimento do intestino e o comprimento total do indivíduo.

Foram estabelecidas as distribuições dos valores médios mensais dos índices de repleção (IR) e hepatossomático (IHS). A determinação da atividade alimentar mensal foi definida a partir do cálculo do índice de repleção, considerando que não foram encontrados estômagos vazios ou parcialmente vazios durante os meses de coleta.

Para análise do conteúdo estomacal foram subamostrados 111 indivíduos, entre machos e fêmeas, com estômagos no estádio de repleção cheio, e com comprimento total variando de 35 a $118 \mathrm{~mm}$. O trato preservado foi aberto em laboratório, sendo os itens alimentares identificados por meio de varredura realizada com auxílio de microscópio, até o menor nível taxonômico possível. O conteúdo foi analisado segundo o método de freqüência de ocorrência (HysLop, 1980). Foi estabelecido um índice de importância alimentar (IIA) para os itens ingeridos, segundo Granado-Lorencio \& Garcia-Novo (1981), baseado em uma escala de abundância semi-quantitativa, de acordo com o número de ocorrência de cada item alimentar em relação ao montante total de itens. Foi adotada a escala: 3, muito abundante (representando mais de $50 \%$ dos itens); 2, abundante (de 25 a $50 \%$ ); 1 , escasso (menos de 25\%). Segundo Guillen \& Granado (1984), considerou-se alimento principal aqueles com valores de IIA acima de 0,3 , alimento adicional de 0,3 a 0,15 e alimento acidental com valores inferiores a 0,15 .

\section{RESULTADOS}

O quociente intestinal médio encontrado para a espécie foi de 9,46, com desvio padrão de 1,48, não havendo uma variação do mesmo ao longo dos diferentes meses do 
ano. Os valores médios dos índices de repleção e hepatossomático (fig. 1) variaram durante os meses de estudo. Observa-se que esses dois índices apresentam uma tendência de variação similar, ocorrendo um período de maior atividade alimentar, compreendendo os meses de maio a julho de 2001. A relação entre os valores destes índices e as variações de pluviosidade e temperatura foi testada através do coeficiente de correlação de Pearson, porém não apresentaram valor significativo.

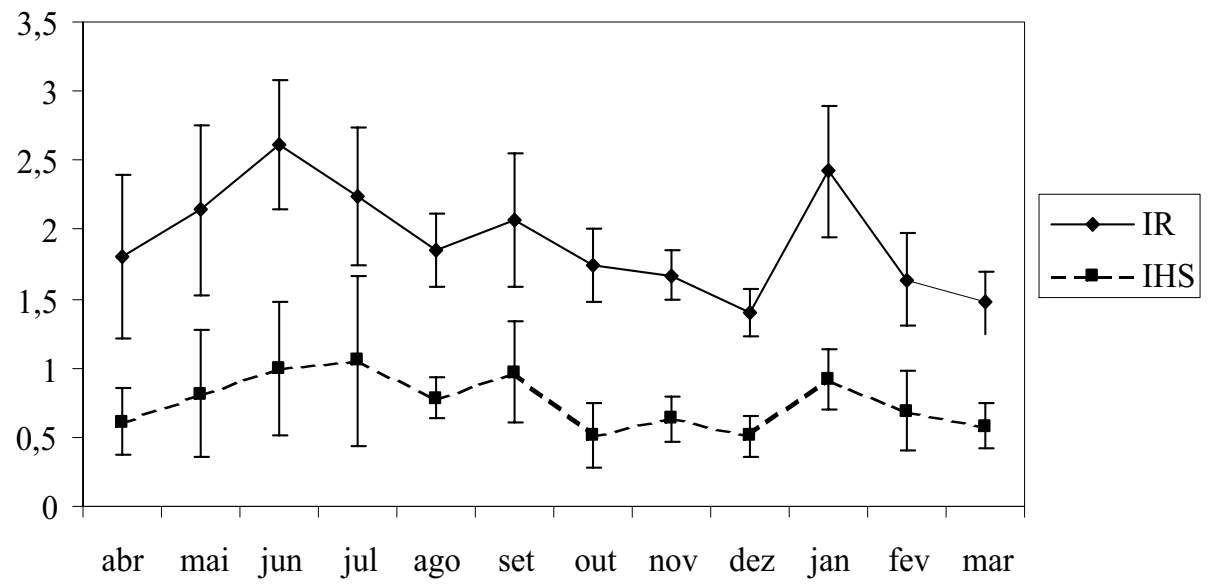

Fig 1. Variação dos valores médios do índice de repleção (IR) e hepatossomático (IHS) para Steindachnerina brevipinna do rio Ibicuí-Mirim, Rio Grande do Sul, no período de abril de 2001 a março de 2002 ( $\mathrm{I}=$ desvio padrão).

Os itens alimentares ingeridos por Steindachnerina brevipinna foram, principalmente, algas pertencentes à divisão Bacillariophyta (diatomáceas), sendo mais freqüente a classe Pennatibacillariophyceae, ordem Pennales, e à divisão Chlorophyta, sendo mais freqüente a família Desmidiaceae (Charophyceae, Zygnematales). Também foram encontrados protozoários compondo os itens alimentares. Segundo o método de freqüência de ocorrência (tab. I), observa-se que as algas diatomáceas formam o item de maior freqüência na alimentação da espécie, ocorrendo em $100 \%$ dos estômagos ao longo de todo o período de estudo. Desmidiaceae também foram muito freqüentes, e as demais algas da divisão Chlorophyta e os Protozoa menos freqüentes.

Tabela I. Distribuição da freqüência relativa de ocorrência dos itens alimentares de Steindachnerina brevipinna do rio Ibicuí-Mirim, Rio Grande do Sul, no período de abril de 2001 a março de 2002.

\begin{tabular}{lcccccccccccc}
\hline Item (\%) & Abr & Mai & Jun & Jul & Ago & Set & Out & Nov & Dez & Jan & Fev & Mar \\
\hline Diatomaceae & 100 & 100 & 100 & 100 & 100 & 100 & 100 & 100 & 100 & 100 & 100 & 100 \\
Desmidiaceae & 80 & 100 & 25 & 100 & 90 & 100 & 80 & 100 & 100 & 100 & 100 & 100 \\
Outras Chlorophyta & 30 & 10 & 50 & 10 & 0 & 0 & 40 & 20 & 40 & 28,5 & 10 & 30 \\
Protozoa & 40 & 50 & 50 & 20 & 10 & 40 & 10 & 20 & 60 & 42,8 & 10 & 0 \\
\hline
\end{tabular}


O índice de importância alimentar (IIA) dos itens ingeridos (tab. II) mostra que as diatomáceas mantiveram-se como alimento principal durante todos os meses do ano, tendo as desmidiáceas variado de alimento principal a adicional (abril e outubro) e acidental em junho. As demais Chlorophyta e os protozoários apresentaram-se, na grande maioria dos meses, como alimento acidental. Não foram observadas diferenças significativas entre os itens ingeridos por machos e fêmeas ou juvenis e adultos.

Tabela II. Índice de importância alimentar (IIA) dos itens alimentares de Steindachnerina brevipinna do rio Ibicuí-Mirim, Rio Grande do Sul, no período de abril de 2001 a março de 2002 (*, alimento adicional; **, alimento acidental).

\begin{tabular}{lllllllllllllll}
\hline & Abr & Mai & Jun & Jul & Ago & Set & Out & Nov & Dez & Jan & Fev & Mar \\
\hline Diatomaceae & 1 & 1 & 1 & 1 & 1 & 1 & 1 & 1 & 1 & 1 & 1 & 1 \\
Desmidiaceae & $0,266^{*}$ & 0,333 & $0,083^{* *}$ & 0,333 & 0,3 & 0,666 & $0,266^{*}$ & 0,333 & 0,333 & 0,666 & 0,333 & 0,333 \\
Outras Chlorophyta & $0,1^{* *}$ & $0,033^{* *}$ & $0,166^{*}$ & $0,033^{* *}$ & $0^{* *}$ & $0^{* *}$ & $0,133^{* *}$ & $0,066^{* *}$ & $0,133^{* *}$ & $0,095^{* *}$ & $0,033^{* *}$ & $0,1^{* *}$ \\
Protozoa & $0,133^{* *}$ & $0,166^{*}$ & $0,166^{*}$ & $0,066^{* *}$ & $0,033^{* *}$ & $0,133^{* *}$ & $0,033^{* *}$ & $0,066^{* *}$ & $0,2^{*}$ & $0,143^{* *}$ & $0,033^{* *}$ & $0^{* *}$ \\
\hline
\end{tabular}

\section{DISCUSSÃO}

Verificou-se, através da análise dos itens alimentares de S. brevipinna, que a mesma apresenta hábito alimentar detritívoro. Corroborando este fato, está o cálculo do quociente intestinal, o qual mostrou-se alto $(9,46)$, significando que o intestino é cerca de 9 vezes o comprimento total do peixe. Segundo Fryer \& ILES (1972), o comprimento do intestino está claramente relacionado à categoria trófica da espécie, sendo ordenada da seguinte maneira: carnívoros < omnívoros $<$ herbívoros $<$ detritívoros. Fugi et al. (2001) encontraram para Steindachnerina insculpta Vari, 1991 o maior comprimento de intestino quando comparado a outras espécies de peixes que se alimentam de fundo, na planície de inundação do rio Paraná. Esta espécie se alimenta exclusivamente de detrito finamente particulado e sedimento. $\mathrm{O}$ intestino longo seria uma adaptação para consumir alimento de difícil digestão e assimilação, que necessita de uma grande área para absorção.

Através da variação dos valores médios dos índices de repleção e hepatossomático, observa-se que ocorreu um aumento destes valores nos meses de maio a julho de 2001 . Esta variação deve estar relacionada à atividade reprodutiva, considerando que nos meses de agosto a dezembro ocorre alta freqüência de indivíduos maduros ou em estádio final de maturação. Antes do período reprodutivo toda a energia obtida através da alimentação seria alocada para o desenvolvimento das gônadas, sendo que, durante a reprodução, haveria apenas uma diminuição da intensidade de alimentação, comprovada pela ausência de estômagos vazios durante o período de estudo. Resultados similares foram encontrados por HARTz et al. (1996) para Oligosarcus jenynsii Günther, 1864 na Lagoa Caconde (RS), e BARBIERI et al. (1982) para Hoplias malabaricus (Bloch, 1794) na Represa do Lobo (SP), cujas espécies também apresentaram maiores freqüências de estômagos cheios antes e após o período reprodutivo sem, no entanto, interromper a atividade alimentar durante o mesmo.

Segundo KaWAKami \& Vazzoler (1980), os índices alimentares determinam a importância efetiva de cada item na alimentação da espécie, combinando freqüência de 
ocorrência e a quantidade observada destes itens que pode ser numérica, volumétrica ou gravitométrica. Não foi possível estimar percentuais em peso ou volume para os itens alimentares, optando-se por uma escala semi-quantitativa, semelhante ao método dos pontos referido por HYSLOP (1980).

Os resultados da análise do conteúdo estomacal demonstram que não há uma variação significativa na qualidade da alimentação ao longo dos meses do ano dado que, unido ao resultado obtido para o quociente intestinal (valor alto, 9,46), denota a alta especialização alimentar de $S$. brevipinna. Fatores abióticos como temperatura, fotoperíodo e pluviosidade foram considerados, porém não apresentaram influência sobre a qualidade alimentar da espécie.

Agradecimentos. Ao Dr. Luiz Roberto Malabarba e aos colegas do Laboratório de Ictiologia (UFRGS) pelo auxílio em campo. Ao CNPq (processo 464545/00-5) pelo financiamento das saídas de campo e aquisição de material.

\section{REFERÊNCIAS BIBLIOGRÁFICAS}

Barbieri, G.; Verani, J. R. \& Barbieri, M. C. 1982. Dinâmica qualitativa da nutrição de Hoplias malabaricus (Bloch, 1974), na Represa do Lobo (Brotas-Itirapina/SP.) (Pisces, Erythrinidae). Revta bras. Biol., Rio de Janeiro, 42(2):295-302.

Bowen, S. H. 1984. Detritivory in neotropical fish comunities. In: ZARET, T. M. Evolutionary ecology of Neotropical freshwater fish. Dordrecht, W. Junk. p.59-66.

Darnell, R. M. 1961. Trophic spectrum of an estuarine community based upon studies of Lake Pontchartrain, Louisiana. Ecology, Temple, 42:552-568.

1964. Organic detritus in relation to secondary production in aquatic communities. Verh. Internat. Verein. Theor. Angew. Limnol., Stuttgart, 15:462-470.

FinK, W. I. \& Fink, S. 1978. A Amazônia Central e seus peixes. Acta Amazonica, Manaus, 8(Supl. 4):19-42.

Fryer, G. \& Iles, T. D. 1972. The cichlid fishes of the great lakes of Africa. Edimburg, Oliver \& Boyd. 230p.

Fugi, R.; Agostinho, A. A. \& Hahn, N. S. 2001. Trophic morphology of five benthic-feeding fish species of a tropical floodplain. Revta bras. Biol., Rio de Janeiro, 61(1):27-33.

Goulding, M. 1980. The fishes and the forest: exploration in Amazonian natural history. Berkeley, University of California. 280p.

Granado-Lorencio, C. \& Garcia-Novo, F. 1981. Cambios ictiologicos durante las primeras etapas de la sucesion en el embalse de Arrocampo (Cuenca del Tajo, Caceres). Boln Inst. Esp. Oceano., Madri, 6:224-243.

Guillen, E. \& Granado, C. 1984. Alimentacion de la ictiofauna del embalse de Torrejon (rio Tajo, Caceres). Limnética, Barcelona, 1:304-310.

Hartz, S. M.; Martins, A. \& Barbieri, M. C. 1996. Dinâmica da alimentação e dieta de Oligosarcus jenynsii (Günther, 1862) na lagoa Caconde, Rio Grande do Sul, Brasil (Teleostei, Characidae). Bolm Inst. Pesca, São Paulo, 23:21-29.

Hyslop, E. J. 1980. Stomach contents analysis; a review of methods and their application. J. Fish Biol., Oban, 17:411-429.

Kawakami, E. \& Vazzoler, G. 1980. Método gráfico e estimativa de índice alimentar aplicado no estudo de alimentação de peixes. Bolm Inst. Oceanogr. S. Paulo, São Paulo, 29(2):205-207.

Roberts, T. 1972. Ecology of fishes in the Amazon and Congo basins. Bull. Mus. comp. Zool., Cambridge, 143:117-147.

Recebido em 26.09.2002; aceito em 02.06.2003. 